\title{
Emulsificantes: atuação como modificadores do processo de cristalização de gorduras
}

\author{
Emulsifiers: acting as modifiers of the crystallization behaviour of fats
}

\section{Camilia Aoyagui dos Santos $^{\mathrm{I}^{*}}$ Chiu Chih Ming ${ }^{\mathrm{I}}$ Lireny Aparecida Guaraldo Gonçalves ${ }^{\mathrm{I}}$}

\section{- REVISÃO BIBLIOGRÁFICA -}

\section{RESUMO}

Os emulsificantes são aditivos empregados com diversas finalidades na produção dos alimentos industrializados, dentre elas para melhorar a textura, o volume, a aeração e a homogeneidade dos produtos. Estudos indicam mais uma função para estes compostos que atuam usualmente estabilizando emulsões, como modificadores do processo de cristalização de óleos e gorduras. Produtos como chocolates, margarinas $e$ sorvetes são elaborados com base nos compostos gordurosos que, ao atingirem a condição de super-resfriamento ou supersaturação, formam cristais e, posteriormente, uma rede cristalina tridimensional, responsável pelos atributos sensoriais dos produtos. Os emulsificantes podem interferir no tempo de indução, na velocidade de formação dos cristais de gordura, além de modificar a morfologia e o hábito cristalino desses cristais, retardando as transições polimórficas indesejáveis e afetando diretamente a qualidade dos produtos. Esta revisão abordou os diferentes efeitos de emulsificantes utilizados em alimentos na cristalização de matérias-primas gordurosas.

Palavras-chave: emulsificantes, ésteres de sorbitana, lecitina, cristalização, polimorfismo.

\section{ABSTRACT}

The emulsifiers are additives used for many purposes in the production of foods, among them to improve texture, volume, aeration and homogeneity of the products. Most studies indicate a new role for this compound that acts usually stabilizing emulsions, as modifier of the crystallization of oils and fats. Products such as chocolate, margarine and ice cream which are made of fats, upon reaching the condition of supercooling or supersaturation, form crystals and then a three-dimensional crystal lattice responsible for sensory attributes of products. The emulsifiers can alter induction time, the rate of formation of fat crystals and modify the morphology and crystal habit of these crystals, retarding undesired polymorphic transitions and affecting quality of products. This review evaluated the effects of different food emulsifiers on the crystallization of fats.

Key words: emulsifiers, sorbitan esters, lecithin, crystallization, polymorphism.

\section{INTRODUÇÃO}

Os alimentos representam sistemas coloidais complexos e a adição de emulsificantes desempenha um papel fundamental para a garantia da textura desejada, da uniformidade do produto e da extensão do seu shelf life (KROG \& SPARSO, 2004; FAERGEMAND \& KROG, 2006). Esses aditivos são regulamentados nos Estados Unidos pelo FDA (Food and Drug Administration) (HASENHUETTL, 1997) e no Brasil pela ANVISA (Agência Nacional de Vigilância Sanitária), de acordo com a resolução CNS/MS no04 de 24 de Novembro de 1988, que estabelece níveis máximos permitidos para cada categoria de alimentos.

As propriedades das redes cristalinas são fundamentais para produtos, como pasta de amendoim, spread, sorvete, margarina, manteiga e chocolate, pois estão relacionadas a muitos atributos sensoriais desejáveis, como espalhabilidade, snap (em chocolates), brilho, textura, dentre outros (NARINE \& MARANGONI, 1999; HARTEL, 2013). Estudos demonstram que os emulsificantes podem modificar o processo de cristalização de gorduras, alterando o hábito cristalino e retardando

IDepartamento de Tecnologia de Alimentos, Faculdade de Engenharia de Alimentos, Universidade Estadual de Campinas (UNICAMP), Avenida Monteiro Lobato, n.80, $1^{\circ}$ andar, CP 6121, 13083-970, Campinas, SP, Brasil. E-mail: camilia.ads@gmail.com.*Autor para correspondência. 
as transições polimórficas indesejáveis (GARTI, 2002; OH et al., 2005; MARTINI \& HERRERA, 2008; RADUJKO et al., 2011). Além disso, em chocolates, podem atuar inibindo o aparecimento do defeito denominado fat bloom, que corresponde à recristalização da gordura, visualmente observada na superfície do produto, promovendo mudanças na morfologia dos cristais (MAHUNGU \& ARTZ, 2002; FAERGEMAND \& KROG, 2006). Segundo GARTI (2002), os emulsificantes poderiam interferir na taxa de cristalização, afetando o número de cristais de gordura formados.

Há poucos estudos que abordam o uso dos emulsificantes como modificadores do processo de cristalização, sendo seus efeitos ainda pouco esclarecidos e mais estudos são necessários para elucidar os mecanismos de sua atuação (GARBOLINO et al., 2005). Em se tratando de uma aplicação recémexplorada, esta revisão objetivou demonstrar o uso desse aditivo como modificador do comportamento de cristalização, através da compilação de estudos que mostram os efeitos da adição dos emulsificantes, usualmente utilizados em alimentos, como os ésteres de sorbitana, os ésteres de sacarose, o poliglicerol polirricinoleato (PGPR) e a lecitina, na cristalização de matérias-primas lipídicas.

\section{Emulsificantes em alimentos}

Os emulsificantes são aditivos funcionais extensivamente utilizados pela indústria de alimentos para melhorar a textura, a estabilidade, o volume, a maciez, a aeração e a homogeneidade, agregando qualidade aos produtos (RADUJKO et al., 2011). Além disso, esses aditivos podem modificar a fase contínua de um produto, conferindo-lhe um efeito específico desejado, como o uso da lecitina em chocolates para reduzir a sua viscosidade e facilitar o seu manuseio (MARTINI \& HERRERA, 2008).

A maioria dos emulsificantes é derivada dos mono e diacilgliceróis ou de álcoois, sendo as classes mais utilizadas em alimentos: os mono e diacilgliceróis, os mono e diacilgliceróis acetilados, os mono e diacilgliceróis fosfatados, os ésteres de propilenoglicol, os ésteres de sorbitana, os ésteres de sacarose, os ésteres de poliglicerol, os ésteres de lactato e a lecitina (HASENHUETTL, 1997; O'BRIEN, 2009).

A estrutura dos emulsificantes é composta por uma parte hidrofílica, que interage com a fase aquosa e outra lipofílica, que interage com a fase oleosa. Essa estrutura permite a sua atuação na interface de duas substâncias imiscíveis. Para esta aplicação em especial, torna-se imprescindível o correto balanço entre a hidrofilicidade e lipofilicidade (HLB) da molécula do emulsificante. Quanto maior o valor do HLB, maior a hidrofilicidade e, quanto menor o valor do HLB, maior a lipofilicidade. Dependendo desse balanço, o aditivo poderá ser usado em emulsões do tipo água em óleo $(\mathrm{A} / \mathrm{O})$ ou óleo em água (O/A) (O’BRIEN, 2009; BASTIDARODRÍGUEZ, 2013).

A parte lipofílica é caracterizada pela presença de uma ou mais cadeias de ácidos graxos, representados pela letra $\mathrm{R}$ nas estruturas químicas da figura 1. Para a molécula de poliglicerol polirricinoleato (PGPR), a Letra R corresponde ao ácido ricinoleico policondensado. Geralmente, são empregados os ácidos graxos saturados de cadeia longa, como o ácido palmítico. Por outro lado, a parte hidrofílica pode corresponder a uma variedade de grupos funcionais, dentre eles, o agrupamento $\mathrm{OH}$, que pode ser visto nas moléculas de ésteres de sorbitana e de ésteres graxos de sacarose, e o agrupamento fosfatídeo nas moléculas dos fosfolipídios, como a lecitina. De acordo com o tipo de grupo polar presente, o emulsificante pode ser classificado em aniônico, catiônico, anfótero e não iônico (HASENHUETTL, 1997).

Nas moléculas dos fosfolipídios, o substituinte (X) (Figura 1) representa os grupos colina, etanolamina, inositol, serina ou glicerol, que se encontram esterificados ao fosfatídeo. O fosfolipídio mais conhecido é a fosfatidilcolina, que compõe a conhecida lecitina (GARTI, 2002).

\section{Cristalização dos óleos e gorduras}

O comportamento de cristalização dos óleos e das gorduras influencia na estabilidade física dos produtos de base gordurosa, no que se refere à exsudação do óleo, à coalescência de glóbulos de gordura em emulsões, à formação e à sedimentação de cristais em um óleo (FOUBERT et al., 2007). Portanto, o entendimento do processo de cristalização de gorduras é fundamental para que a gordura cumpra eficientemente o seu papel no produto final, garantindo sua qualidade (HIMAWAN et al., 2006).

Segundo HARTEL (2013), a cristalização de um sistema no estado líquido ocorre quando se atinge o estado de supersaturação, em que as moléculas se unem para formarem núcleos estáveis. Após a formação desses núcleos, há a etapa denominada crescimento dos cristais, que se prolonga até o sistema atingir o equilíbrio termodinâmico.

A combinação da nucleação e do crescimento dos cristais possibilita a formação de uma rede contínua de cristais, responsável pelas 


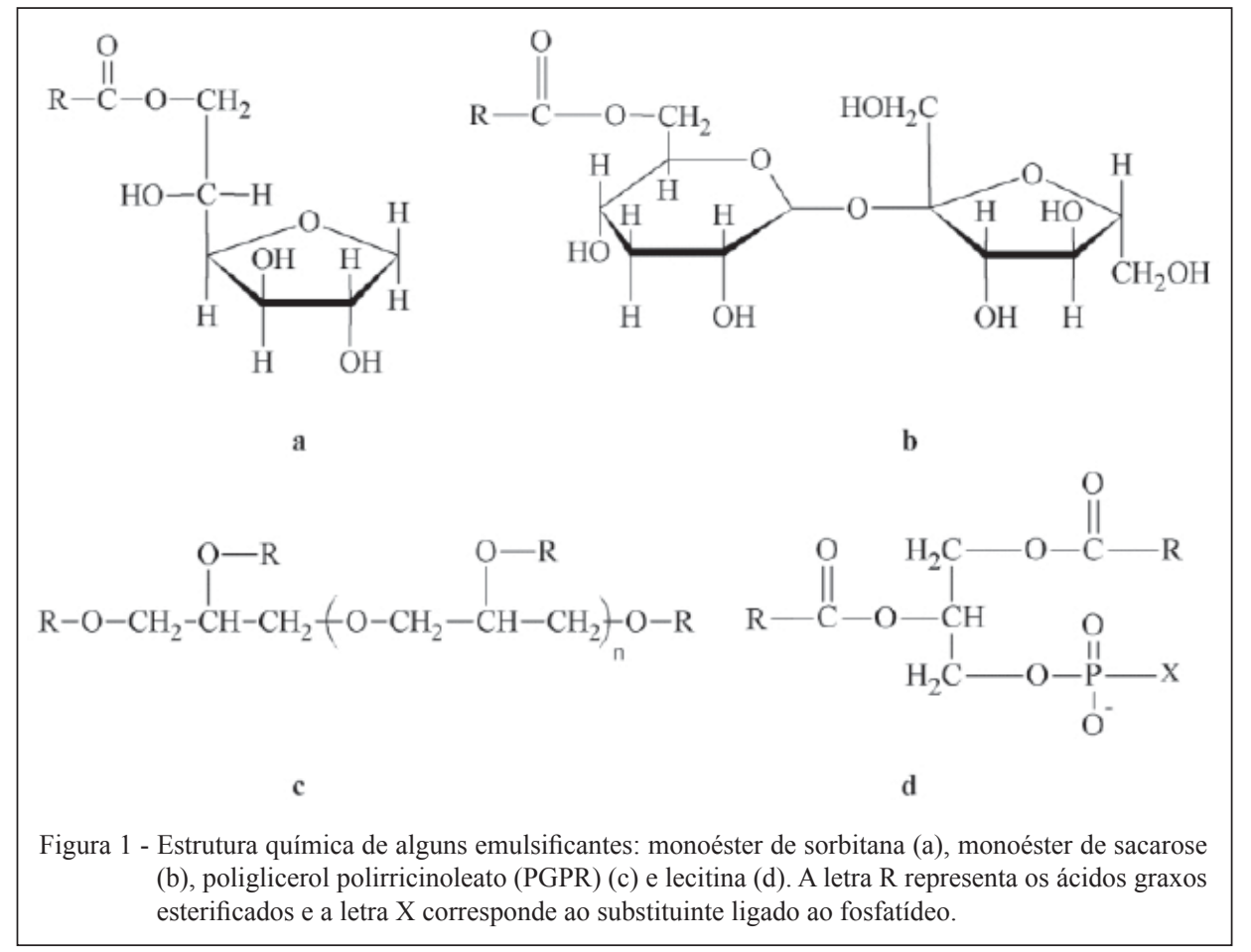

propriedades macroscópicas e mecânicas de uma gordura (FOUBERT et al., 2007).

A equação mais utilizada na literatura para descrever a cinética de cristalização isotérmica de gorduras é o modelo de Avrami (MARANGONI, 2005; FOUBERT et al., 2007; MENG et al., 2011; RIBEIRO et al., 2012), que tem a seguinte forma:

$$
\frac{S F C}{S F C m \mathbf{a} x}=1-e^{-k t^{n}}
$$

Em que: $t$ indica o tempo do processo; SFC ou CGS representa o conteúdo de gordura sólida em relação ao tempo; $\mathrm{SFC}_{\text {máx }}$ ou $\mathrm{CGS}_{\text {máx }}$ indica o teor máximo de gordura sólida atingido; $\mathrm{k}$ corresponde à constante da taxa de cristalização e $n$ refere-se ao índice de cristalização e indica o mecanismo de crescimento dos cristais (MARANGONI, 2005).

Os cristais podem se apresentar em distintas formas polimórficas, denominadas de $\alpha$, $\beta^{\prime}$ e $\beta$. Essas formas são baseadas nas estruturas de subcélulas oriundas do empacotamento das cadeias alifáticas das moléculas dos triacilgliceróis, presentes nos óleos e nas gorduras (SATO, 2001). Uma forma polimórfica menos estável como a forma $\alpha$ pode ser convertida na forma de estabilidade intermediária, $\beta^{\prime}$, e esta para a forma mais estável $\beta$, sem ocasionar qualquer mudança na estrutura química dos cristais (O’BRIEN, 2009, SATO \& UENO, 2011).
Em margarinas e em shortenings, podem existir duas formas polimórficas, $\beta^{\prime}$ e $\beta$, sendo os cristais com forma $\beta^{\prime}$ os desejáveis para esses tipos de produtos, por serem relativamente pequenos, por incorporarem uma grande quantidade de matéria graxa líquida na rede cristalina, proporcionando ao produto final uma superfície brilhante, textura lisa sem granulosidade e com uma boa espalhabilidade (DEMAN \& DEMAN, 2002; ROUSSEAU et al., 2005; SATO \& UENO, 2011).

Na manteiga de cacau, o comportamento polimórfico é complexo. Essa matéria-prima se cristaliza em 6 formas polimórficas, identificadas comumente como I, II, III, IV, V e VI, sendo a forma VI a mais estável (WILLE \& LUTTON, 1966). No entanto, há muitas divergências na literatura principalmente quanto à nomenclatura. A forma $\mathrm{V}$, ou comumente denominada como $\beta \mathrm{V}$, é a forma desejada para os chocolates e é produzida na etapa de temperagem (COHEN et al., 2004; SVANBERG et al., 2011).

Atuação dos emulsificantes como modificadores do processo de cristalização

De acordo com GARTI (2002), durante o processo de cristalização, a interação entre as moléculas dos emulsificantes e dos triacilgliceróis pode ocorrer de 3 formas distintas, de acordo com a miscibilidade entre elas: miscibilidade limitada, quando o emulsificante age como impureza, podendo promover ou retardar o crescimento dos cristais e as 
transições polimórficas; alta miscibilidade ou total imiscibilidade, em que o emulsificante age como gérmen de cristalização, favorecendo a nucleação.

Outros autores consideram que os triacilgliceróis e os emulsificantes seriam capazes de co-cristalizarem, devido à similaridade das suas estruturas químicas. Além disso, em geral, quanto maior a dissimilaridade entre eles e quanto maior a massa molar do emulsificante, maior seria o seu potencial inibidor no processo de cristalização (KATSURAGI, 1999).

\section{Ésteres de sorbitana}

Os efeitos dos ésteres de sorbitana na cristalização de triacilgliceróis foram inicialmente determinados em sistemas modelo (MOHAMED \& LARSSON, 1992; ELISABETTINI et al., 1995), nos quais foi comprovada a influência desses aditivos, principalmente no retardo das transições polimórficas. Posteriormente, foram realizados estudos envolvendo o efeito desses emulsificantes em sistemas reais, como os óleos e gorduras, que apresentam uma mistura complexa de triacilgliceróis.

Um éster de sorbitana bastante conhecido é o triestearato de sorbitana (STS). A adição de 5\% $(\mathrm{m} / \mathrm{m})$ desse emulsificante à manteiga de cacau promoveu a transição polimórfica da forma $\beta^{\prime}$ para a forma $\beta \mathrm{V}$ e retardou totalmente a transição de $\beta \mathrm{V}$ para $\beta \mathrm{VI}$, possibilitando uma manteiga de cacau com presença somente do polimorfo $\beta \mathrm{V}$ (SMITH et al., 2011; TALBOT et al., 2012). Essa forma polimórfica é desejável em chocolates à base de manteiga de cacau, pois a sua faixa de temperatura de fusão é próxima à temperatura corporal, garantindo completa fusão na boca.

Estudos apontam que, durante o armazenamento dos chocolates à base de manteiga de cacau, a mesma transição polimórfica, da forma $\beta \mathrm{V}$ para a forma $\beta \mathrm{VI}$, seria a responsável pelo aparecimento do defeito visual, denominado fat bloom (TALBOT, 2009) e, como a adição de STS impediu o seu aparecimento (FAERGEMAND \& KROG, 2006; O'BRIEN, 2009), pode-se concluir que a sua atuação residiria no controle do polimorfismo.

O mesmo efeito no retardo da transição polimórfica da forma $\beta \mathrm{V}$ para a forma $\beta \mathrm{VI}$ foi verificado após a adição de $5 \%(\mathrm{~m} / \mathrm{m})$ de monoestearato ou de monopalmitato de sorbitana na manteiga de cacau, proporcionando uma importante aplicação desta classe de emulsificantes no controle das transições polimórficas indesejáveis (SMITH et al., 2011).

O STS também se mostrou capaz de modificar beneficamente o conteúdo de gordura sólida (CGS). A adição de $0,03 \% \quad(\mathrm{~m} / \mathrm{m})$ deste emulsificante em amostras contendo misturas de óleo de palma refinado e oleína de palma, nas proporções 60:40, 50:50 e 30:70, acarretou o aumento do valor do CGS medido a $20^{\circ} \mathrm{C}$, demonstrando o efeito do emulsificante em acelerar a cristalização (MISKANDAR, 2006).

Outra matéria-prima bastante utilizada na indústria de alimentos é o óleo de palma, cuja cristalização pode ser considerada lenta e complexa. Problemas como a formação de arenosidade pósprocessamento sempre foram motivos de preocupação da indústria de margarinas e shortenings que utilizam o óleo de palma como matéria-prima. Estudos indicam que o aparecimento dessa arenosidade seria ocasionada pela flutuação da temperatura no armazenamento do produto, com a consequente transição polimórfica dos triacilgliceróis (POP) da forma $\beta^{\prime}$ para a forma $\beta$ e formação de cristais de dimensões maiores $(>20 \mu \mathrm{m})$ (WATANABE et al., 1992; OMAR et al., 2005).

GARBOLINO et al. (2005) demonstraram, através da análise da microestrutura dos cristais, formada após a cristalização (sob oscilação térmica) de uma mistura contendo $20,6 \%$ de óleo de palma, $29,4 \%$ da mistura interesterificada de óleo de palma e óleo de palmiste e $50 \%$ de óleo de girassol, que a adição de $2 \%(\mathrm{~m} / \mathrm{m})$ de monopalmitato ou de monoestearato de sorbitana evitou a recristalização, responsável pela formação de grânulos, indicando uma aplicação promissora na produção de margarinas à base de óleo de palma. $\mathrm{O}$ mesmo efeito não foi alcançado com o uso de monolaurato de sorbitana, evidenciando que o tamanho da cadeia de ácido graxo presente no emulsificante pode resultar em efeitos diversos no comportamento de cristalização dos triacilgliceróis, corroborando com GARTI (2002) e MING et al. (2012).

Além disso, a comparação dos efeitos dos ésteres de sorbitana na cristalização de óleos e gorduras entre os diferentes estudos pode ser dificultada devido à variabilidade encontrada na composição desses emulsificantes. Esses aditivos podem apresentar proporções distintas nas suas formas mono-, di- e triésteres (GARTI et al., 1983), apesar de serem comercializados usualmente sob a denominação de monoésteres ou triésteres. Além disso, é geralmente empregada uma mistura de ácidos graxos de diferentes tamanhos de cadeia na reação de formação desses ésteres de sorbitana. O triestearato de sorbitana (STS) comercial, por exemplo, pode constituir apenas de 1 a $3 \%$ do triéster de ácido esteárico (SMITH et al., 2011; TALBOT et al., 2012). 
Ésteres de sacarose

Em estudo com os ésteres de sacarose, CERDEIRA et al. (2005) concluíram que a adição de $0,1 \%(\mathrm{~m} / \mathrm{m})$ de estearato ou de palmitato de sacarose nas misturas contendo a fração lipídica de alto ponto de fusão do leite e óleo de girassol atrasou o tempo de indução (nucleação) e o crescimento dos cristais, prejudicando a cristalização. De acordo com PUPPO et al. (2002), esse resultado poderia ser explicado pela ocorrência da co-cristalização dos emulsificantes com os triacilgliceróis. No entanto, no estudo de GARBOLINO et al. (2005), os mesmos ésteres de sacarose, adicionados na concentração de $2 \%(\mathrm{~m} / \mathrm{m})$ em uma mistura à base de óleo de palma, óleo de girassol e óleo de palmiste, aceleraram a cristalização, verificada pelo aumento substancial no número de cristais de pequenas dimensões. Além disso, os autores, contrariamente ao estudo de PUPPO et al. (2002), apontaram a eficaz cocristalização, promovida pela grande similaridade do tamanho de cadeia entre o palmitato de sacarose e os ácidos palmítico e oleico, predominantes no óleo de palma, como fator responsável para a aceleração da cristalização.

Resultado similar ao observado por GARBOLINO et al. (2005) foi obtido após adição dos ésteres de sacarose em uma emulsão água em óleo $(\mathrm{A} / \mathrm{O})$ à base de óleo de palmiste, que, de acordo com os autores, agiram como gérmens de cristalização, acelerando a nucleação (AWAD \& SATO, 2002). Esses efeitos adversos dos emulsificantes demonstram que ainda não foram totalmente esclarecidos os mecanismos de atuação desses aditivos no comportamento de cristalização de sistemas reais, que apresentam uma mistura de triacilgliceróis.

OH \& SWANSON (2006) determinaram os efeitos da adição de $5 \%(\mathrm{~m} / \mathrm{m})$ de poliésteres de sacarose, dentre eles, os poliésteres de ácidos esteáricos com diferentes valores do balanço entre hidrofilicidade e lipofilicidade (HLB), na transição polimórfica de $\beta \mathrm{V}$ para $\beta \mathrm{VI}$ de manteiga de cacau. Os autores observaram que, quanto menor o valor de HLB (maior lipofilicidade) e, portanto, maior quantidade de poliésteres presentes, maior foi o retardo na referida transição polimórfica, o que pode representar uma aplicação vantajosa desses emulsificantes na produção de chocolates, em vista da estabilidade da forma $\beta \mathrm{V}$, que é a forma desejada neste tipo de produto. Segundo os autores, o efeito no retardo das transições polimórficas seria devido à eficiente co-cristalização das moléculas do aditivo com os triacilgliceróis majoritários na manteiga de cacau, que apresenta o ácido esteárico dentre os ácidos graxos predominantes na composição.

Em estudo prévio, $\mathrm{OH}$ et al. (2005) já haviam demonstrado o efeito dos estearatos de sacarose em retardar a transição polimórfica de $\alpha$ ou $\beta$ ' para $\beta$ de triestearina (SSS). De acordo com o estudo, os ésteres de sacarose com ácidos graxos de tamanho de cadeia semelhante ao dos ácidos graxos presentes na triestearina e de menor valor de HLB (maior lipofilicidade) foram os que apresentaram maior potencial inibidor das transições polimórficas.

\section{Poliglicerol polirricinoleato}

O poliglicerol polirricinoleato (PGPR) é outro emulsificante muito utilizado em alimentos, principalmente para reduzir o Casson yield value, alterando a viscosidade em chocolates e facilitando a sua moldagem (HASENHUETTL, 1997; LONCHAMPT \& HARTEL, 2004; FAERGEMAND \& KROG, 2006; BASTIDA-RODRÍGUEZ, 2013). Poucos estudos exploraram a aplicação do PGPR como modificador do comportamento de cristalização. Um destes estudos é o de ROUSSEAU et al. (2005), que demonstraram que o uso de $0,125 \%(\mathrm{~m} / \mathrm{m})$ de PGPR na cristalização da mistura de óleo de algodão hidrogenado e óleo de canola, realizada a $5^{\circ} \mathrm{C}$ e sob agitação, retardou a transição polimórfica da forma $\beta^{\prime}$ para $\beta$.

O PGPR também é capaz de reduzir a incidência do fat bloom em chocolates. Esse defeito pode ter origem em diversos fatores, dentre eles a recristalização da manteiga de cacau e as transições polimórficas que podem ocorrer durante estocagem, principalmente, quando há oscilação de temperatura (BASTIDA-RODRÍGUEZ, 2013). Possivelmente, o emulsificante agiria nas transições polimórficas da manteiga de cacau, alterando o seu comportamento de cristalização. Outra influência, relatada na literatura, seria a capacidade do PGPR em aumentar substancialmente a velocidade de cristalização da manteiga de cacau, possibilitando um menor intervalo de tempo necessário para a cristalização completa de chocolates (BOWSER, 2006).

\section{Lecitina}

A lecitina, assim como o PGPR, é muito utilizada na produção de chocolates, tanto para o controle da viscosidade quanto para o controle do fat bloom (JEFFERY, 1991; HASENHUETTL, 1997; LONCHAMPT \& HARTEL, 2004). Ainda poucos estudos apontaram a lecitina como modificador do comportamento de cristalização.

DHONSI \& STAPLEY (2006) estudaram o efeito da adição de $0,2 \%(\mathrm{~m} / \mathrm{m})$ de lecitina na 
mistura contendo manteiga de cacau e açúcar durante a pré-cristalização. Os autores verificaram que o emulsificante reduziu sutilmente o tempo de indução ou nucleação, acelerando o processo de cristalização da manteiga de cacau. Em outro estudo, resultado similar foi obtido. Durante a cristalização isotérmica da estearina do óleo de palmiste hidrogenado nas temperaturas de $15,20,25$ e $30^{\circ} \mathrm{C}$, a adição de lecitina e de monoestearato de sorbitana permitiu a redução do tempo de indução dos cristais nas temperaturas de 25 e $30^{\circ} \mathrm{C}$ (WANG et al., 2011).

De acordo com SAVAGE \& DIMICK (1995), as moléculas de fosfatidilcolina, que compõem a lecitina, devem apresentar uma configuração hexagonal inversa durante a cristalização da manteiga de cacau considerada "hard". Esta conformação seria totalmente favorável para a interação com as moléculas de triacilgliceróis, em uma possível cocristalização, o que explicaria os tempos de indução menores e as altas taxas de crescimento dos cristais na presença do fosfolipídio.

Outra explicação para a atuação da lecitina na presença de outros componentes do chocolate, além da manteiga de cacau, seria a de que este fosfolipídio reduziria a interação entre os sólidos do cacau e a gordura, permitindo à gordura o livre acesso para formar as estruturas cristalinas, o que promoveria a cristalização (JEFFERY, 1991).

Além desse efeito no tempo de indução, a lecitina poderia atuar como gérmen de cristalização na manteiga de cacau, dando início ao processo de formação dos cristais. A velocidade de crescimento dos cristais de gordura na amostra aditivada foi superior à velocidade verificada na amostra não aditivada (SVANBERG et al., 2011).

\section{CONCLUSÃO}

Os emulsificantes de uso em alimentos podem não somente ser utilizados para melhorar a textura, a estabilidade, o volume, a maciez, a aeração e a homogeneidade dos produtos, como também atuar na modificação do comportamento de cristalização dos óleos e gorduras, matérias-primas que compõem a maior parte dos alimentos. Esses aditivos agem reduzindo o tempo de indução (nucleação), aumentando a taxa de crescimento dos cristais e impedindo a formação de cristais de dimensões maiores, perceptíveis sensorialmente, reduzindo a incidência do defeito visual fat bloom em chocolates, retardando as transições polimórficas indesejáveis, agregando rapidez e qualidade aos produtos de base gordurosa.
No entanto, esses efeitos na cristalização podem ser distintos e inclusive antagônicos, dependendo do perfil de ácidos graxos e de triacilgliceróis do óleo ou gordura onde o emulsificante foi adicionado, da concentração utilizada e da composição do emulsificante. Os mecanismos que explicam as interações entre as diferentes estruturas moleculares dos emulsificantes e os triacilgliceróis ainda não estão completamente esclarecidos e mais estudos precisam ser conduzidos.

\section{AGRADECIMENTOS}

Os autores agradecem ao Conselho Nacional de Desenvolvimento Científico e Tecnológico $(\mathrm{CNPq})$ e à Fundação de Amparo à Pesquisa do Estado de São Paulo (FAPESP), pelo apoio financeiro ao projeto.

\section{REFERÊNCIAS}

AWAD, T.; SATO, K. Acceleration of crystallisation of palm kernel oil in oil-in-water emulsion by hydrophobic emulsifier additives. Colloids and Surfaces Biointerfaces, v.25, p.45-53, 2002. Disponível em: <http://ac.els-cdn.com/S0927776501002983/1s2.0-S0927776501002983-main.pdf?_tid=00ebc3ee-b4eb-11e2b332-00000aab0f27\&acdnat $=1367693400 \_238245909 \mathrm{~d} 762 \mathrm{f} 8 \mathrm{c} 07$ 933eb4f8032563>. Acesso em: 02 maio 2013.

BASTIDA-RODRÍGUEZ, J. The food additive polyglycerol polyricinoleate(E-476): structure, applications, andproductionmethods. ISRN Chemical Engineering, v.2013, p.1-21, 2013. Disponível em: $<$ http://www.hindawi.com/isrn/chemeng/2013/124767/>. Acesso em 20 abr. 2013. doi: 10.1155/2013/124767.

BOWSER, A. Crystallization of cocoa butter. Manufacturing Confectionary, v.86, n.9, p-115-118, 2006.

BRASIL. Resolução CNS/MS nº 04 de 24 de Novembro de 1988. Aprova a tabela de aditivos intencionais. Disponível em: $<$ http://portal.anvisa.gov.br/wps/wcm/connect/2d98d4804745761 d8413d43fbc4c6735/Resolucao_04_1988.pdf?MOD=AJPERES $>$. Acesso em: 10 maio 2013.

CERDEIRA, M. et al. Nucleation behavior of blended highmelting fractions of milk fat as affected by emulsifiers. European Journal of Lipid Science and Technology, v.107, p.877885, 2005. Disponível em: <http://onlinelibrary.wiley.com/ doi/10.1002/ejlt.200500257/pdf>. Acesso em: 05 set. 2011. doi: 10.1002/ejlt.200500257.

COHEN, K.O. et al. Temperagem ou pré-cristalização do chocolate. Brazilian Journal of Food Technology, v.7, n.1, p.2330, 2004. Disponível em: <http://bjft.ital.sp.gov.br/artigos/html/ busca/PDF/v7nu158a.pdf>. Acesso em: 05 abr. 2013.

DEMAN, J.M.; DEMAN, L. Texture of fats. In: MARANGONI, A.G.; NARINE, S.S. Physical properties of lipids. New York: Marcel Dekker, 2002. Cap.7.

DHONSI, D.; STAPLEY, A.G.F. The effect of shear rate, temperature, sugar and emulsifier on the tempering of cocoa butter. Journal of Food Engineering, v.77, p.936-942, 2006. Disponível em: $<$ http://ac.els-cdn. com/S026087740500573X/1-s2.0-S026087740500573X-main.pdf? 
tid $=8 \mathrm{e} 05 \mathrm{c} 3 \mathrm{~d} 4-4 \mathrm{f} 91-11 \mathrm{e} 2-8 \mathrm{ddb}-00000 \mathrm{aacb} 360 \& \mathrm{acdnat}=1356549914$ fdcb2e161dd1ce1983eac148d3ab78f2>. Acesso em: 12 jun. 2011. doi: 10.1016/j.jfoodeng.2005.08.022

ELISABETTINI, P. et al. Effect of sorbitan tristearate on the thermal and structural properties of monoacid triglycerides influence of a "cis" or " "trans" double bond. European Journal of Lipid Science and Technology, v.97, n.2, p.65-69, 1995. Disponível em: <http://onlinelibrary.wiley.com/doi/10.1002 lipi.19950970206/pdf>. Acesso em: 20 abr. 2013. doi: 10.1002/ lipi.19950970206.

FAERGEMAND, M.; KROG, N. Interactions of emulsifiers with other components in foods. In: GAONKAR, A.G.; MCPHERSON, A. Ingredients interactions - effects on food quality. 2.ed. Boca Raton: Taylor \& Francis Group, 2006. Cap.12, p.390-421.

FOUBERT, I. et al. Physical properties: structural and physical characteristics. In: GUNSTONE, F.D. et al. The lipid handbook. 3.ed. Boca Raton: CRC, 2007. p.471-490.

GARBOLINO, C. et al. The influence of emulsifiers on the crystallization behavior of a palm oil-based blend. Europe Journal Lipid Science Technology, v.107, n.9, p.616-626, 2005. Disponível em: <http://onlinelibrary.wiley.com/doi/10.1002/ejlt.200501186/ pdf>. Acesso em: 11 set. 2011. doi: 10.1002/ejlt.200501186.

GARTI, N. et al. Analysis of sorbitan fatty acid esters by HPLC. Journal of American Oil Chemists' Society, v.60, n.6, p.11511154, 1983. Disponível em: <http://link.springer.com/content/ pdf/10.1007\%2FBF02671346.pdf>. Acesso em: 12 abr. 2013. doi: $10.1007 / \mathrm{BF} 02671346$

GARTI, N. Food emulsifiers: structure-reactivity, relationships, design, and applications. In: MARANGONI, A.G.; NARINE, S. Physical properties of lipids. New York: Marcel Dekker, 2002. Cap.9, p.265-386.

HARTEL, R. W. Advances in Crystallization in Foods. Annual Review of Food Science and Technology, v.4, p.277-292, 2013 Disponível em: <http://www.annualreviews.org/doi/pdf/10.1146 annurev-food-030212-182530>. Acesso em: 10 nov. 2013. doi: 10.1146/annurev-food-030212-182530.

HASENHUETTL, G.L. Overview of food emulsifiers. In: HASENHUETTL, G.L.; HARTEL, R.W. Food emulsifiers and their applications. New York: Chapman \& Hall, 1997. Cap.1, p.1-26.

HIMAWAN, C. et al. Thermodynamic and kinetic aspects of fat crystallization. Advances in Colloid and Interface Science, v.122, p.3-33, 2006. Disponível em: <http://ac.els-cdn.com/ S0001868606000923/1-s2.0-S0001868606000923-main.pdf? tid=f57dbade-599c-11e2-82bf-00000aab0f6c\&acdnat=1357654324 a04d76c8a39aa449bb70db722b4259ae>. Acesso em: 31 out. 2012. doi: 10.1016/j.cis.2006.06.016.

JEFFERY, M.S. The effect of cocoa butter origin, milk fat and lecithin levels on the temperability of cocoa butter systems. Manufacturing Confectionary, v.71, n.6, p.76-82, 1991 .

KATSURAGI, T. Interactions between surfactants and fats. In: WIDLAK, N. Physical properties of fats, oils and emulsifiers. Champaign: AOCS, 1999. Cap.12, p.211-219.

KROG, N.J.; SPARSO, F.V. Food emulsifiers: their chemical and physical properties. In: FRIBERG, S.E. et al. Food emulsions. 4.ed. New York: Marcel Dekker, 2004. Cap.2.
LONCHAMPT, P.; HARTEL, R.W. Fat bloom in chocolate and compound coatings. European Journal of Lipid Science and Technology, v.106, p.241-274, 2004. Disponível em: <http:// onlinelibrary.wiley.com/doi/10.1002/ejlt.200400938/pdf>. Acesso em: 04 dez. 2011. doi: 10.1002/ejlt.200400938.

MAHUNGU, S.M.; ARTZ, W.E. Emulsifiers. In: BRANEN, A.L. et al Food additives. 2.ed. New York: Marcel Dekker, 2002. Cap.23.

MARANGONI, A.G. Crystallization kinetics. In: MARANGONI, A.G. Fat crystal networks. New York: Marcel Dekker, 2005. Cap.2, p.21-82.

MARTINI, S.; HERRERA, M.L. Physical properties of shortenings with low-trans fatty acids as affected by emulsifiers and storage conditions. European Journal of Lipid Science and Technology, v.110, p.172-182, 2008. Disponível em: <http://onlinelibrary.wiley. com/doi/10.1002/ejlt.200700196/pdf`. Acesso em: 30 jul. 2012. doi: 10.1002/ejlt.200700196

MENG, Z. et al. Comparative analysis of lipid composition and thermal, polymorphic, and crystallization behaviors of granular crystals formed in beef tallow and palm oil. Journal of Agricultural and Food Chemistry, v.59, p.1432-1441, 2011. Disponível em: $<$ http://pubs.acs.org/doi/pdfplus/10.1021/jf103875f $>$. Acesso em: 20 maio 2013. doi: $10.1021 / \mathrm{jf} 103875 f$.

MING, C.C. et al. Effect of sorbitan esters composition on crystallization of refined palm oil. In: EURO FED LIPID CONGRESS, 10, 2012, Cracow, Poland. Online only supplement - book of abstracts. New Jersey: Wiley-Blackwell, 2012. v.114. 460p. p.332.

MISKANDAR, M.S. et al. Effects of emulsifiers on crystallization properties of low-melting blends of palm oil and olein. Journal of Food Lipids, v.13, n.1, p.57-72, 2006. Disponível em: <http:// onlinelibrary.wiley.com/doi/10.1111/j.1745-4522.2006.00034.x/ pdf>. Acesso em: 25 maio 2012. doi: 10.1111/j.17454522.2006.00034.x.

MOHAMED, H.M.A.; LARSSON, K. Effects on phase transitions in tripalmitin due to the presence of dipalmitin, sorbitan-monopalmitate or sorbitan-tripalmitate. European Journal of Lipid Science and Technology, v.94, n.9, p.338-341, 1992. Disponível em: <http:// onlinelibrary.wiley.com/doi/10.1002/lipi.19920940905/pdf>. Acesso em: 06 abr. 2013. doi: 10.1002/lipi.19920940905.

NARINE, S.S.; MARANGONI, A.G. Relating structure of fat crystal networks to mechanical properties: a review. Food Research International, v.32, n.4, p.227-248, 1999. Disponível em: <http:// ac.els-cdn.com/S0963996999000782/1-s2.0-S09639969990000782main.pdf? tid=e7a36c60-5992-11e2-a930-00000aab0f01\&acdnat= 1357650006_07144d78093b3024a0437550b3ec9755>. Acesso em: 16 jan. 2012. doi: 10.1016/S0963-9969(99)00078-2.

O'BRIEN, R.D. Fats and oils formulation. In: O'BRIEN, R.D. Fats and oils - formulating and processing for applications. 3.ed. Boca Raton: CRC, 2009. Cap.4, p.263-345.

$\mathrm{OH}$, J. et al. Stabilizing polymorphic transitions of triestearin using diacylglycerols and sucrose polyesters. Journal of American Oil Chemists' Society, v.82, p.13-19, 2005. Disponível em: <http:// link.springer.com/content/pdf/10.1007\%2Fs11746-005-1036-y>. Acesso em: 04 jul. 2011. doi: 10.1007/s11746-005-1036-y.

OH, J.H.; SWANSON, B.G. Polymorphic transitions of cocoa butter affected by high hydrostatic pressure and sucrose polyesters. 
Journal of American Oil Chemists' Society, v.83, p.1007-1014, 2006. Disponível em: <http://link.springer.com/content/pdf/10 .1007\%2Fs11746-006-5155-2>. Acesso em: 26 jun. 2012. doi: $10.1007 / \mathrm{s} 11746-006-5155-2$.

OMAR, Z. et al. Crystallization and rheological properties of hydrogenated palm oil and palm oil blends in relation to crystal network. European Journal of Lipid Science and Technology, v.107, p.634-640, 2005. Disponível em: < http://onlinelibrary. wiley.com/doi/10.1002/ejlt.200501180/pdf>. Acesso em: 05 abr. 2013. doi: 10.1002/ejlt.200501180.

PUPPO, M.C. et al. Effects of sucrose esters on isothermal crystallization and rheological behavior of blends of milkfat fraction sunflower oil. Journal of Food Science, v.67, n.9, p.3419-3426, 2002. Disponível em: <http://onlinelibrary.wiley. com/doi/10.1111/j.1365-2621.2002.tb09600.x/pdf>. Acesso em: 04 maio 2013. doi: 10.1111/j.1365-2621.2002.tb09600.x.

RADUJKO, I. et al. The influence of combined emulsifier 2 in 1 on physical and crystallization characteristics of edible fats. European Food Research and Technology, v.232, n.5, p.899904, 2011. Disponível em: <http://link.springer.com/content/pdf/ 10.1007\%2Fs00217-011-1458-0>. Acesso em: $01 \mathrm{dez}$. 2012. doi: $10.1007 / \mathrm{s} 00217-011-1458-0$.

RIBEIRO,A.P.B. et al. Physico-chemical properties of Brazilian cocoa butter and industrial blends. Part II - Microstructure, polymorphic behavior and crystallization characteristics. Grasas y Aceites, v.63, n.1, p.89-99, 2012. Disponível em: <http://grasasyaceites.revistas. csic.es/index.php/grasasyaceites/article/view/1358/1355>. Acesso em: 14 abr. 2013. doi: 10.3989/gya.069111.

ROUSSEAU, D. et al. Regulating the $\beta^{\prime} \rightarrow \beta$ polymorphic transition in food fats. Journal of American Oil Chemists' Society, v.82, p.7-12, 2005. Disponível em: <http://link.springer. com/article/10.1007\%2Fs11746-005-1035-z?LI=true\#page-1>. Acesso em: 19 jul. 2012. doi: 10.1007/s11746-005-1035-z.

SATO, K. Crystallization behaviour of fats and lipids - A review. Chemical Engineering Science, v.56, p.2255-2265, 2001 Disponível em: <http://ac.els-cdn.com/S0009250900004589/1s2.0-S0009250900004589-main.pdf?_tid=a7339436-aa12-11e29a34-00000aacb35d\&acdnat=1366500966 778bd77a4f2e2e3403 1dfa6bb2873460>. Acesso em: 10 abr. $201 \overline{3}$.

SATO, K.; UENO, S. Crystallization, transformation and microstructures of polymorphic fats in colloidal dispersion states. Current Opinion in Colloid \& Interface Science, v.16, n.5, p.384-390, 2011. Disponível em: <http://ac.els-cdn.com/ S135902941100080X/1-s2.0-S135902941100080X-main.pdf? tid=708591d6-a919-11e2-81e0-00000aacb35d\&acdnat=1366393 930_7ce83dc4e7230ec6644a501fe2715896>. Acesso em: 13 abr. 2013. doi: 10.1016/j.cocis.2011.06.004.

SAVAGE, C.M.; DIMICK, P.S. Influence of phospholipids during crystallization of hard and soft cocoa butters. Manufacturing Confectionary, v.75, n.5, p.127-132, 1995.

SMITH, K.W. et al. Crystallization of fats: influence of minor components and additives. Journal of American Oil Chemists' Society, v.88, n.8, p.1085-1101, 2011. Disponível em: <http:// link.springer.com/article/10.1007\%2Fs 11746-011-18197?LI=true\#page-1>. Acesso em: 02 out. 2011. doi: 10.1007/ s11746-011-1819-7.

SVANBERG, L. et al. Effect of sugar, cocoa particles and lecithin on cocoa butter crystallisation in seeded and non-seeded chocolate model systems. Journal of Food Engineering, v.104, p.70-80, 2011. Disponível em: <http://ac.els-cdn.com/S0260877410005777/1s 2.0-S $0260877410005777-$ main.pdf?_tid=5d 5 acaf6ac46-11e2-8211-00000a acb361\&acdnat=1366743079 e6502dd6b3f579320ea141f4b2e02d92>. Acesso em: 18 abr. $2013^{-}$. doi: 10.1016/j.jfoodeng.2010.09.023.

TALBOT, G. Chocolate temper. In: BECKETT, S.T. Industrial chocolate manufacture and use. 4.ed. Oxford: Blackwell publishing, 2009. Cap.12, p.261-275.

TALBOT, G. etal. Influence of minor components on fat crystallization. Lipid Technology, v.24, n.4, p.83-85, 2012. Disponível em: <http:// onlinelibrary.wiley.com/doi/10.1002/lite.201200180/pdf>. Acesso em: 23 abr. 2013. doi: 10.1002/lite.201200180.

WANG, F. et al. Kinetic analysis of isothermal crystallization in hydrogenated palm kernel stearin with emulsifier mixtures. Food Research International, v.44, p.3021-3025, 2011. Disponível em: <http://ac.els-cdn.com/S0963996911004546/1s2.0-S0963996911004546-main.pdf? tid=083 dbdfc-4a1d-11e2be03-00000aab0f02\&acdnat $=1355950112 \_3677 \mathrm{a} 9 \mathrm{~d} 8 \mathrm{e} 5 \mathrm{a} 8874 \mathrm{f}$ 0eae8078dfd66042>. Acesso em: 20 ago. 2012. doi: 10.1016/j. foodres.2011.07.014.

WATANABE, A. et al. On the formation of granular crystals in fat blends containing palm oil. Journal of American Oil Chemists' Society, v.69, p.1077-1080, 1992. Disponível em: <http://link. springer.com/content/pdf/10.1007\%2FBF02541040>. Acesso em: 11 abr. 2013. doi: 10.1007/BF02541040.

WILLE, R.L., LUTTON, E.S. Polymorphism of cocoa butter. Journal of the American Oil Chemists' Society, v.43, p.491-496, 1966. Disponível em: <http://link.springer.com/article/10.1007\%2 FBF02641273?LI=true\#page-1>. Acesso em: 22 maio 2012. doi: $10.1007 / \mathrm{BF} 02641273$ 